\title{
EDITORIAL
}

(2)

\section{Advancing drug therapy of the metabolic syndrome}

\author{
The metabolic syndrome - a group of factors that raise the risk of cardiovascular disease \\ and type 2 diabetes - is increasingly common. How might the challenges of developing \\ improved therapies to normalize these risk factors be approached?
}

The metabolic syndrome is a rapidly growing public health burden. Obesity is considered to be the driving force, because it can elicit all the defining cardiometabolic risk factors: atherogenic dyslipidaemia, increased plasma glucose levels, increased blood pressure, a pro-thrombotic state and a pro-inflammatory state. Consequently, firstline therapy for the metabolic syndrome is lifestyle change: that is, weight reduction and increased physical activity. Unfortunately, lifestyle therapy can be difficult to implement in practice, and the risk factors are subject to other influences, such as genetic predispositions. So, to effectively normalize the risk factors of the metabolic syndrome, it is often necessary to use drugs.

However, at present, the only available such approach is to use separate drugs to target each risk factor. Moreover, some risk factors, such as hyperglycaemia in type 2 diabetes, may themselves require multiple drugs to achieve adequate control. Although this approach has the benefit of allowing a physician to titrate and monitor the efficacy of each drug for each risk factor, the disadvantages include greater risk of adverse drug interactions, complicated treatment regimens and high cost. There has therefore been increasing interest in alternative strategies to develop drug therapies that could positively affect several risk factors.

One strategy is the 'polypill', whereby several risk factors are treated with a single capsule containing a combination of drugs, which can be assembled in various ways. Although the polypill cannot be titrated for better risk factor control when used alone, its advantages include simplicity and cost reduction if generic drugs are used. In a recent promising Phase II trial of such a polypill, the once-daily formulation contained a generic statin, low doses of three types of generic antihypertensive drug and aspirin ${ }^{1}$. Such a polypill might be used as a public health measure for the whole population to reduce the risk of cardiovascular events, as an alternative to universal lifestyle modification ${ }^{2}$. Another potential broad use is for secondary prevention in patients with established cardiovascular disease in developing countries, where the high costs of conventional therapies are prohibitive.

A second pharmacological strategy to reduce the problems that are associated with polypharmacy for patients with several risk factors is to develop single drugs that have multiple targets or modulate targets that affect several risk factors ${ }^{3}$. For example, the angiotensin II receptor blocker telmisartan lowers blood pressure through its action on the renin-angiotensin system and also improves dysglycaemia through inherent activity at the peroxisome proliferator-activated receptor (PPAR)- $\gamma$. However, in general, this approach to the treatment of multiple cardiometabolic risk factors is at an early stage and poses considerable challenges, as illustrated by the history of the dual PPARa-PPAR $\gamma$ modulator muraglitazar. This potential diabetes drug was intended to combine the established beneficial effects of PPARa modulation on dyslipidaemia and of PPAR $\gamma$ modulation on hyperglycaemia, but concern over its cardiovascular safety led to the discontinuation of its development after Phase III trials.

Of course, the two approaches above to treat multiple cardiometabolic risk factors also share a common challenge: the need to demonstrate efficacy and safety in large, heterogeneous populations. At present, it is possible to achieve regulatory approval on the basis of targeting specific risk factors such as high blood pressure, high cholesterol levels and increased blood glucose levels, but not a nonspecific clustering of risk factors. Although some single-pill combinations of registered drugs (such as a statin for high cholesterol levels and a $\mathrm{Ca}^{2+}$ channel blocker for hypertension) have recently been approved by the US FDA, regulatory agencies seem to be setting a higher bar for new anti-diabetic agents and lipidlowering drugs. So, it looks likely that the evolution of regulatory standards will also play an important part in advancing the therapy of the metabolic syndrome beyond the current approach of polypharmacy.

Scott M. Grundy

1. The Indian Polycap Study (TIPS). The effects of a polypill (Polycap) on risk factors in middle-aged individuals without cardiovascular disease (TIPS): a phase II, double-blind, randomised trial. Lancet 30 Mar 2009 (doi:10.1016/S0140-6736(09)60611-5).

2. Wald, N. J. \& Law, M. R. A strategy to reduce cardiovascular disease by more than $80 \%$. BMJ 326,1419 (2003).

3. Grundy, S. M. Drug therapy of the metabolic syndrome: minimizing the emerging crisis in polypharmacy. Nature Rev. Drug Discov. 5 , 295-309 (2006). 